\title{
Deriving Software Design Models from a Set of Business Processes
}

\author{
Estrela F. Cruz ${ }^{1,2}$, Ricardo J. Machado ${ }^{2}$ and Maribel Y. Santos ${ }^{2}$ \\ ${ }^{1}$ Instituto Politécnico de Viana do Castelo, Viana do Castelo, Portugal \\ ${ }^{2}$ Centro ALGORITMI, Escola de Engenharia, \\ Universidade do Minho, Guimarães, Portugal
}

Keywords: $\quad$ Business Process Modeling, BPMN, Use Case Model, Data Model, UML.

\begin{abstract}
Requirements elicitation is a crucial activity and one of the first steps in software development process. A popular way to capture and describe requirements is through UML use case models. Transforming requirement specifications into software design models is a complex and error prone software development activity. Software design usually involves several models, each one representing a different perspective. One of those perspectives is the data perspective which can be modeled using a data model. Although of the importance of this model, few works has been done to derive a data model from use case model. The 4SRS (4-Step Rule Set) method generates a logical architecture of a software-based system, based on a use case model. This paper proposes an approach to adapt and extend the 4SRS method in order to generate a data model supporting the generated logical architecture and the elicited requirements based in a set of business process models.
\end{abstract}

\section{INTRODUCTION}

In the software development process, one of the first activities is the identification of the system scope and the understanding of what the system is supposed to do. Some software development teams base the entire software development process on the list of requirements, identified in the first stage of the development process and modeled using use case models. Another key point in software development is the transformation of the requirements specification, modeled as use cases, into software design models. This is mainly because, at this stage, the problem specification starts to be transformed into a software product solution (Bragança and Machado, 2006). That is, in fact, the main goal of the 4SRS (4 Step Rule Set) method. The 4SRS method produces a logical architecture from the user requirements, represented as use cases (Santos and Machado, 2010; Machado et al., 2006). It employs successive transformations of the software architecture in order to satisfy the elicited requirements. This method is especially useful to make the transition from requirements modeled as use cases, to the architecture of large and complex software systems (Ferreira et al., 2012), because in these cases the possibility to lose or forget some detail is high and it is very complex to manage the use cases. The $4 \mathrm{SRS}$ method enables us to prevent these problems, but to do so it requires a use case model with a high detail level. For this, the decomposition triangle approach, presented in (Cruz et al., 2014c), may be used helping in decomposing and refining use cases to achieve a high detail level.

In software development, different models are usually used to represent different perspectives. The data model is one of the most important design models for building software applications, representing and organizing data, how it is stored and accessed, and the relationships among different entities.

The business process management is being increasingly used by organizations as a means to improve their products quality and to improve their productivity. With the aim of assuring the alignment between business processes and software requirements, the approaches presented in (Cruz et al., 2014b; Cruz et al., 2015) generate a use case model including use case descriptions based on the information available in a set of interrelated business process models. In the approach being presented here, we intend to use this generated use case model as input to the 4SRS generating the logical architecture and corresponding data model, thus supporting the set of business process models. The approach presented in this paper adapts and extends the 4SRS in order to generate the data model. This way it will be possible to generate the software system logical architecture and their supporting data model.

The remainder of this paper is structured as fol- 
lows. Next section summarizes the 4SRS method and briefly presents the language used to specify use case descriptions. Section 3 presents the Nobel Prize example which is used as a demonstration case. Section 4 describes the proposed approach for data model identification. In section 5 some related work is presented. Finally, conclusions and some remarks to future work are presented in section 6.

\section{BACKGROUND}

A software logical architecture represents the software system main components and the relation between them, allowing to understand the organization of the system. The 4SRS method ensures the transition from user requirements specified as use case models into software logical architectures, objects and class models. A complete description of the 4SRS method can be found in (Machado et al., 2006).

Nowadays, use case models are one of the most common models used in requirements modeling and elicitation. A use case diagram is composed by actors and use cases. Each use case shall have an associated description. There are some alternatives that can be used to describe a use case, such as informal text, numbered steps or pseudo-code (Cockburn, 2001). The approach presented in (Cruz et al., 2014b) proposes a template simplifying the Cockburn's template, for use cases description. To fill out the use case description template, a set of structured sentences is created in Natural Language (NL). The template and the set of sentences were originally designed to support the automatic generation of the use case model and corresponding descriptions based on the existing information in a BPMN model (Cruz et al., $2014 b$ ), or a set of BPMN models as described in (Cruz et al., 2015). The set of sentences covers all possible actions, flows and graphical elements present in a BPMN model. Files (or documents) produced or received by an activity ("transported" by a data association or a message flow) are attached to the corresponding use case descriptions.

Sentences involving data and that, for that reason, are relevant for deriving a data model, are listed next:

- Writes information on $<$ data store name $>$.

- Reads information from $<$ data store name $>$.

- Sends/Receives $<$ document name $>$.

- Receives $<$ message name $>$ from $<$ actor name $>$.

- Sends $<$ message name $>$ to $<$ actor name $>$.

The complete set of sentences that can be used can be found in (Cruz et al., 2014b). In this context, a use case represents a business process activity (Cruz et al., 2014b). Pre-conditions are used to constrain the use cases execution order.

In this paper, the 4 SRS method is adapted to deal with use case models using a structured and controlled language in use case descriptions and is extended to generate the data model (section 4).

\section{NOBEL PRIZE EXAMPLE}

In this section we present, as a demonstration case, a well-known and small example of the Nobel Prize.

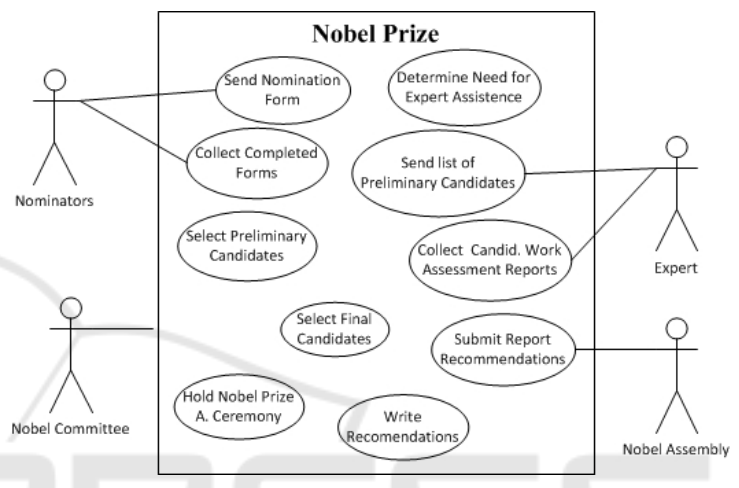

Figure 1: Nobel Prize use case diagram.

The use case diagram generated from the original business process model, as described in (Cruz et al., 2014c), is shown in Figure 1. The corresponding use case descriptions are presented in Table 1. Due to lack of space only the descriptions of use cases considered most important are presented. All identified use cases are numbered using the tag=value UML mechanism.

The next section describes the approach proposed in this paper to obtain a data model by adapting and extending the 4SRS method. This Nobel Prize example is used throughout the next section as a running example.

\section{DERIVING A LOGICAL ARCHITECTURE AND THE SUPPORTING DATA MODEL}

In the approach presented herein we intend to generate the data model based on the information available in a use case model, where each use case description is created using a set of structured NL sentences previously defined (Cruz et al., 2014b).

The original 4SRS method is organized in 4 steps transforming use-case models into architectural ele- 
Table 1: The descriptions of the use cases using the defined template.

\begin{tabular}{|c|c|}
\hline $\begin{array}{l}\text { Use case } \\
\text { Name }\end{array}$ & Use case Description \\
\hline $\begin{array}{l}\{\mathrm{U} 1\} \text { Send } \\
\text { Nomination } \\
\text { Form }\end{array}$ & $\begin{array}{l}\text { Actors: Nobel Committee, Nominator } \\
\text { Trigger: The time-date September is reached. } \\
\text { Scenario: Read information from } \\
<\text { Nominator }>\text {. Around } 3000 \text { invitations } \\
\text { confidential nomination forms are sent to } \\
\text { selected Nominators. Sends the Nomination } \\
\text { Invitation to }<\text { Nominator }>\text {. }\end{array}$ \\
\hline $\begin{array}{l}\{\mathrm{U} 2\} \\
\text { Collect } \\
\text { Completed } \\
\text { forms }\end{array}$ & $\begin{array}{l}\text { Actors: Nobel Committee, Nominator } \\
\text { Pre-condition: Send Nomination Form has } \\
\text { been completed. } \\
\text { Scenario: Receives nomination Form from } \\
<\text { Nominator }>\text {. Write information on } \\
<\text { Nominator }>\text {. }\end{array}$ \\
\hline $\begin{array}{l}\{\mathrm{U} 3\} \text { Select } \\
\text { Preliminary } \\
\text { Candidates }\end{array}$ & $\begin{array}{l}\text { Actors: Nobel Committee } \\
\text { Pre-condition: Collect Completed forms have } \\
\text { been completed. } \\
\text { Scenario: Read information from } \\
<\text { Nominator }>\text {. Write information on } \\
<\text { Candidates }>\text {. }\end{array}$ \\
\hline $\begin{array}{l}\{\text { U5\}Send } \\
\text { List of } \\
\text { Preliminary } \\
\text { Candidates }\end{array}$ & $\begin{array}{l}\text { Actors: Nobel Committee, Expert } \\
\text { Pre-condition: The Expert Assistance Re- } \\
\text { quired? is Yes. } \\
\text { Scenario: Read information from } \\
<\text { [Preliminary }] \text { Candidates }>\text {. Sends the } \\
\text { List of Candidates to be Assessed to } \\
<\text { Expert }>\text {. }\end{array}$ \\
\hline $\begin{array}{l}\{\text { U6\}Collect } \\
\text { assessment } \\
\text { Report }\end{array}$ & $\begin{array}{l}\text { Actors: Nobel Committee, Expert } \\
\text { Pre-condition: Send List of Preliminary } \\
\text { Candidates has been completed. } \\
\text { Scenario: Receives assessments from } \\
\text { <Expert }>\text {. Write information on } \\
\text { <Assessment }>\text {. }\end{array}$ \\
\hline $\begin{array}{l}\{\text { U } 7\} \text { Select } \\
\text { Final Can- } \\
\text { didates }\end{array}$ & $\begin{array}{l}\text { Actors: Nobel Committee } \\
\text { Pre-condition: Collect assessment Report has } \\
\text { been completed. } \\
\text { Scenario: Read information from } \\
\text { <Assessment }>\text {. }\end{array}$ \\
\hline $\begin{array}{l}\{\text { U8 }\} \text { Write } \\
\text { Recommen- } \\
\text { dations }\end{array}$ & $\begin{array}{l}\text { Actors: Nobel Committee } \\
\text { Pre-condition: The Expert Assistance Re- } \\
\text { quired? is No OR Select Final Candidates } \\
\text { has been completed. } \\
\text { Scenario: Write information on } \\
\text { <Recommendations }>\text {. }\end{array}$ \\
\hline $\begin{array}{l}\{\text { U9 }\} \text { Submit } \\
\text { Report Rec- } \\
\text { ommenda- } \\
\text { tions }\end{array}$ & $\begin{array}{l}\text { Actors: Nobel Committee, Nobel Assembly } \\
\text { Pre-condition: Write Recommendations has } \\
\text { been completed. } \\
\text { Scenario: Read information from } \\
<\text { Recommendations }>\text {. Sends Recom- } \\
\text { mendations to }<\text { Nobel Assembly }>\text {. }\end{array}$ \\
\hline
\end{tabular}

ments (Machado et al., 2006). The 4SRS method executes a series of validations and adjustments to the original use case model. In order to deal with structured sentences and allowing the generation of a data model, the original 4SRS steps are slightly modified as presented next:

- Step 1 - Architectural element creation: in this step, the original 4SRS method proposes the creation of three types of objects for each use case: one interface, one data and one control. However, we are able to distinguish between persistent from non-persistent data, as it happens in the BPMN language (OMG, 2011). Following this idea, the 4SRS will be adapted to also distinguish persistent data from non-persistent data, by creating two different types of elements involving data: persistent data and volatile data. Each element is labeled with the name of the use case followed by the appropriate type: $i$ (interface), $c$ (control), $d p$ (data persistent) and $d v$ (data volatile). This step can be automated since it does not involve decisions.

- Step 2 - Architectural element elimination: based on the textual description of each use case, it is necessary to decide which of the four elements, created in step 1, must be maintained. This step allows detecting and eliminating redundancy in requirements. This step is divided into seven micro-steps:

- Step $2 \mathrm{i}$ - Use cases classification: In this micro step each use case is classified as interface, data persistent, data volatile, control, or any combination of these. This classification aims to facilitate the transformation of each use case in architectural elements as it provides clues about which categories of elements to use and how they are related. Since we are dealing with a set of structured sentences in use case descriptions, it is possible to classify the use case following the suggestions:

* When a use case exchanges information with something or someone (usually an actor), the $i$-interface type must be selected. One use case represents the interaction with an external participant (represented as an actor) using sentences like Receives $<$ message name $>$ from <actor name $>$ or Sends <message name $>$ to $<$ actor name $>$.

* When information is stored or retrieved then the type $d p$-data persistent must be selected. Information is stored or retrieved when sentences like Writes information on $<$ data store name $>$ or Reads information from $<$ data store name $>$ are part of the use case descrip- 
tion.

* When in a use case description we have sentences like Receives <document name $>$ or Sends $<$ document name $>$, this means that we are dealing with non-persistent data, thus the type $d v$-data volatile must be selected. It is natural to find non-persistent data transformed in persistent data because in a business process, most of the times, data received from a participant is then stored in a data store.

* When a use case description has a trigger, a pre-condition or a post-condition, then the type $c$-control must be selected.

Taking as example the $\{U 6\}$ - Collect assessment Report use case (from Table 1) we may see that the use case is receiving non-persistent data (assessments) from expert (Receives assessments from Expert), meaning that it is interacting with someone, so this is classified as $i$-interface and as $d v$-data volatile. Reading the use case descriptions we may also see that we are dealing with persistent data because the use case Writes information on $<$ Assessment $>$, so the use case is also classified as $d p$-data persistent. The use case has also controlling activities if Send List of Preliminary Candidates has been completed. So, the use case is also classified as c-control. Summarizing, this use case will be classified as $c, i, d v$ and $d p$.

- Step 2ii: Local elimination - the purpose of this micro step is to check if each architectural element created in step 1 makes sense for the problem domain. Those that do not make sense should be eliminated.

- Step 2iii: Architectural element naming - Each architectural element created, should receive a name fitting its original use case as well as the role that it has in the system.

Taking as example the $\{06-d p\}$-Collect assessment Report element created in $\{U 6\}$ - Collect assessment Report use case, we may rename it to $\{06-d p\}-$ Write Assessment to better fit the element purpose.

- Step 2iv: Architectural element description Each architectural element that received a name in previous micro-step should be described according to the corresponding system requirements, in order to be included in the logic model (depicted by objects diagram).

Taking as example the $\{06-d p\}$ - Write Assessment element created in $\{U 6\}$-Collect assessment Report use case, the corresponding description may be the sentence belonging to the original description which leads to the element classification as dp-persistent data. The sentence is Writes information on Assessment. The other sentences will lead to the other types of elements. This is a manual step, so the software architect may complement the description with other information every time it is necessary. At this software development stage, the stakeholders are still involved in the process so they can provide useful information to complement the information generated based on business process models.

- Step 2v: Architectural element representation - This micro-step, through an analysis of each element, ensures the semantic consistency of the logic model, detects and eliminates redundancy, and enables the discovery of anomalies in use case models, namely missing requirements.

Taking as example the $\{U 1\}$-Send Nomination Form and the $\{U 3\}$ - Select Preliminary Candidates use cases, we may see that both have the same $d p$ element ( $d p$-Read Nominator) because both are reading information from $<$ Nominator $>$. As such, both elements can be represented by the same $d p$ architectural element.

- Step 2vi: Global elimination - In this step all micro architectural elements that are represented by other architectural elements are eliminated, since the requirements that correspond to these architectural elements no longer belong to them.

Continuing the example presented in previous micro-step where we conclude that the $\{01 . d p\}$ Read Nominator can be represented by $\{03 . d p\}$ Read Nominator architectural elements or viceversa, one of them must be eliminated.

- Step 2vii: Architectural elements renaming This micro step aims to rename all the remaining architectural elements.

Sometimes, when we have one architectural element, representing several architectural elements (micro-step 2v), the name of this element may be renamed to better fit its purpose.

- Step 3: Architectural elements aggregation and packaging - the architectural elements that remain after the elimination, and those in which it is possible and exist advantages in their unification, are aggregated; At this step, architectural elements that have similar characteristics and can be treated in an unified way are aggregated in the same package.

For example, architectural elements representing persistent data manipulation can be package in 
P3 - Database Control. Architectural elements representing controlling actions are packaged in $P 2$ - Business rules and elements representing user interface are packaged in $P 1$ - User Interface. The Pl - User Interface package may be divided in several sub-packages representing interactions with specific actors. As such, architectural elements representing interactions with Nominator actor are packaged in P1.1 - UI Nominator. Architectural elements representing interactions with Expert actor are packaged in P1.1 - UI Expert and architectural elements representing interactions with Nobel Assembly are packaged in P1.1 - UI Nobel Assembly (see Figure 2).

- Step 4: Architectural elements association - associations must link the elements resulting from the aggregation based on use cases textual descriptions.

The logical architecture resulting from the application of the 4SRS to the Nobel Prize example, is represented in Figure 2.

To define a persistent data model one needs to identify the domain entities, their attributes, and the relationships $((1: n),(m: n)$ or $(1: 1))$ between entities (Weske, 2012). Therefore, the 4SRS will be extended with three additional steps, which are:

- Step 5: Entities creation - in this step, the entities involved in each use case are identified.

- Step 6: Relationships identification - in this step, the relationship between the entities identified in step 5 are identified.

- Step 7: Entity attributes identification - in this step, the attributes belonging to each entity are identified.

Each one of the newly added steps is explained next.

\section{Step 5 - Entities Creation}

An entity is something identifiable, or a concept in the real world that is important to the modeling purpose (Weske, 2012). To identify the entities, this step is divided into two micro-steps, explained next:

- Micro-step 5i: Entities identification - Focusing on the $\{-d p\}$ - persistent data architectural elements remaining in the generated logical architecture, each read, written or updated element gives origin to an entity in the resulting data model.

Looking at the generated logical architecture from the Nobel Prize example, represented in Figure 2, we may see that we are reading and writing information in Nominator, Candidates, Assessment and Recommendations thus, each one of these gives origin to an entity in the data model.

- Micro-step 5ii: Entities representation - This step produces the final list of entities, by detecting and removing the repeated entities. It is usual to find use cases reading information that is written (or updated) by other use cases, especially when we are working with a large number of use cases. As such, it is very common to identify (in the previous micro-step) the same entity several times. These duplicated entities are eliminated.

Backing to the Nobel Prize example and looking to the generated logical architecture (represented in Figure 2), in $\{$ O2.dp $\}$ - Write Nominator architectural element, the entity Nominator is identified. The same entity is also identified in $\{01 . d p\}$ and $\{$ O3.dp $\}$ - Read Nominator architectural element.

The Candidates entity may be identified in $\{03 . d p\}$ - Write Candidates and in $\{05 . d p\}$ Read Candidates architecture elements. Both entities are represented by the Candidates entity. Similar reasoning may be used in Assessment and Recommendations entities.

\section{Step 6 - Relationships Identification}

A relationship between two entities is represented through an association between those entities (Chen, 1976). The role of an entity in a relationship is the function that it executes in that relationship. A relationship between two entities can be classified according to two aspects, Cardinality and Optionality. Both terms are used to denote the number of attributes in a relation. Cardinality represents the maximum number of instances (one or many) of an entity in relation to another entity. Relationship optionality represents the minimum number of elements that exist on that side of the relationship. It may be 1 (the relation is mandatory) or 0 (the relation is not mandatory).

Focusing on the elements, remaining in the resulting logical architecture, that store information (elements with name starting by write or update), we must verify in which conditions the information is stored. When an $\{-d p\}$ - persistent data element is related with a $\{-c\}$ - control element that verifies the information about another entity, we may conclude that the information stored is related with the information checked. Usually, this verification is done by reading information already stored. As a consequence, the entity that represents the written information is related with the entity that represents the checked (and read) information. The relationship is $(1: n)$ from the entity that represents the information checked (previ- 


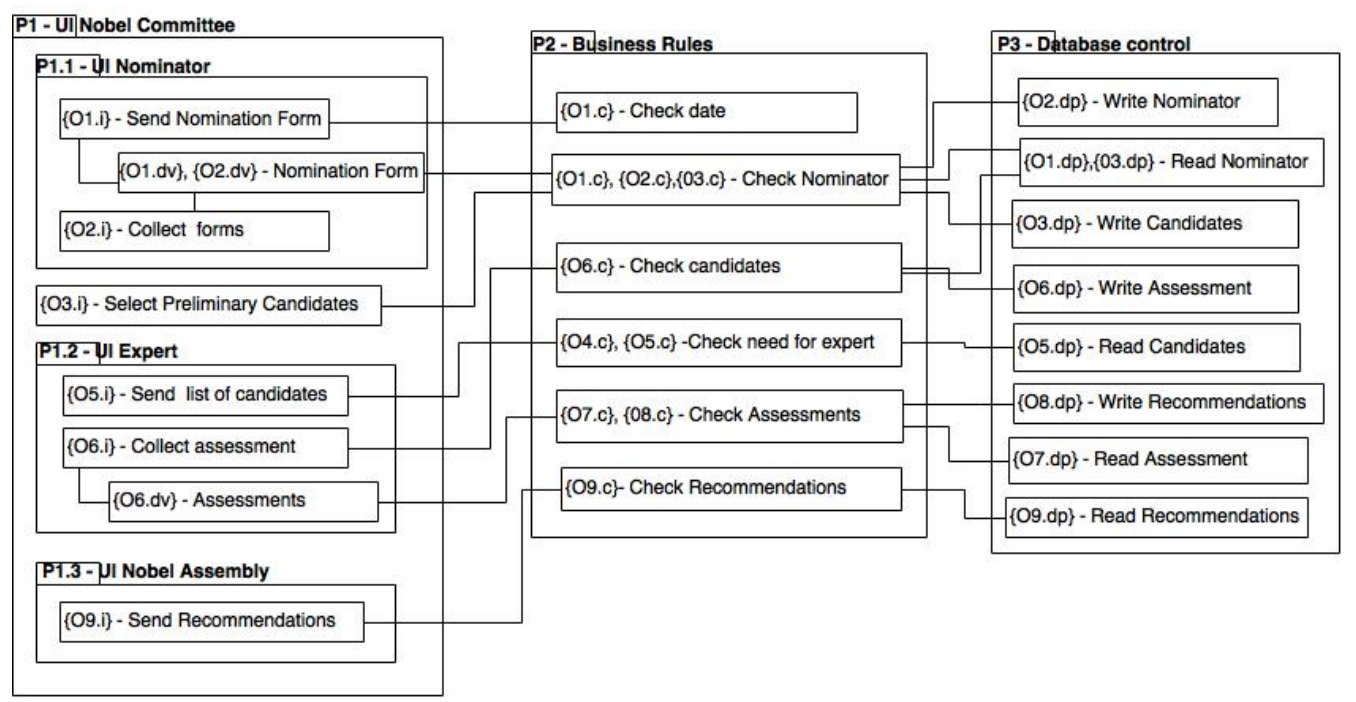

Figure 2: The Nobel Prize resulting logical architecture.

ously written information) to the entity that represents the written information because the same information can be read several times and associated to different written information items. On the other hand the information is stored only once.

By default the relationship is mandatory on the side of the information checked (the information must be verified) and is not mandatory on the side of the written entity because the information may be written, or not, depending on the verification result.

Looking to the $\{06 . d p\}$ - Write Assessments element we may see that it is related with $\{06 . c\}$ - Check Candidates, so we may conclude the Assessments entity is related with the Candidates entity. The same happens between Candidates and Nominator and between Recommendations and Assessments. The relationship is (1:n) from the read to the written entity and not mandatory from the side of the written entity.

\section{Step 7 - Entity Attributes Identification}

The information, or the properties, about an entity are expressed through a set of attributes (Weske, 2012). Since we are dealing with use cases which descriptions are generated from business process models, in some cases to prevent model complexity the properties are not identified. Nevertheless, in some cases, especially when information is stored or retrieved, the use case description may have a document in attachment (Cruz et al., 2014b) originated from attachments in BPMN data elements or messages. The document may identify items stored or retrieved. In that case, each item represents an entity attribute. In cases where the properties are not identified, the software architect may ask for more detailed information, complementing this way the information generated from the business process models. In the 4SRS method, most of the steps are manual and some of them require the software architect expertise (Machado et al., 2006). At this software development process phase, the stakeholders are still involved and available to provide answers.

The resulting data model from the Nobel Prize example is shown in Figure 3. In the resulting data model, we are using the following syntax for each relationship end. Focusing in one side of a relationship type and considering the optionality and cardinality together we have: 0 or 1 (represented as to-), 1 ( $\Pi$ ), 0 to many $(-\infty)$ ) and 1 to many $(-k)$.

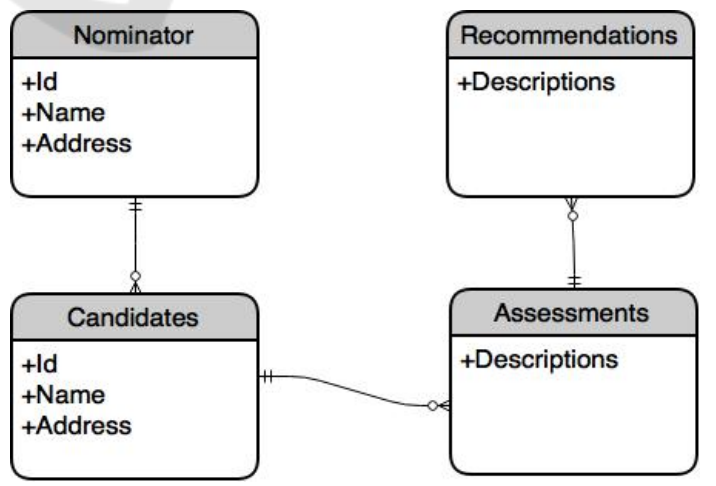

Figure 3: The Nobel Prize resulting data model.

Through micro-step $5 i$ we are able to identify the entities Nominator, Candidates, Assessments and Recommendations representing data stored.

Applying step 6, we are able to identify relationships between: Nominator and Candidates, Candi- 
dates and Assessments, Assessments and Recommendations. As previously explained, the relationships are (1:n) from first to the second entity and the relationship is mandatory on the side of the first entity and not mandatory on the other side.

\section{RELATED WORK}

In the software development process many models are used to represent different points of view. Some models, like use case models, may have information that can be used to generate other software models. Nevertheless most of that information is in use case descriptions, commonly specified in NL (Fantechi et al., 2003; Cockburn, 2001). NL may be easy to understand but, at the same time, can be ambiguous, redundant and with omissions (Fantechi et al., 2003). The approach presented herein is dealing with a set of controlled sentences previously defined in NL facilitating an automated analysis. The set of sentences are defined with the purpose of transforming business process models (BPMN) into use case models (Cruz et al., 2014b).

Model transformation is one of the basic principles of Model Driven Architecture (Yue et al., 2011). Several authors propose approaches to derive software models (data models and class models) from requirements representations (use case models). Some of the existing approaches are presented next.

Samarasinghe and Som propose an approach to create a Domain model from a Use Case model (Samarasinghe and Somé, 2005). To describe use cases the authors use a controlled NL.The authors state that automatic processing of use cases described in full NL is not possible so, they propose a restricted form of NL grammar for use case descriptions (Samarasinghe and Somé, 2005).

In (Ilieva and Ormandjieva, 2006) the authors propose the generation of the domain model and UML activity diagram from requirements specification. The authors consider unlimited NL, but they rewrite it in a different format using a tabular presentation of the text. They start with a syntactical analysis of the text, and then build a tabular presentation and a semantic network. The construction of models are conducted in four stages: syntax categorization, tabular modeling of the text, semantic processing of the text, and interpretation of the text for diagrammatic modeling (Ilieva and Ormandjieva, 2006).

Yue et al. (Yue et al., 2009) to avoid NL ambiguity, propose a set of restriction rules and a new template to describe use cases. The authors aim is to facilitate the textual analysis, allowing the automatic extraction of the UML class model. The restriction rules and the template are to be used during the requirements elicitation phase.

All surveyed existing approaches try to restrict the NL used in use case descriptions. The approach presented herein also uses a restricted NL but specially designed to represent the information we have in business process models. Therefore, the approach uses a set of sentences previously defined with the aim of generating use cases from one or more Business process models, modeled in BPMN. The generation of the sentences is prepared to be automatic as well as the construction of the 4SRS tabular transformation and its first step.

\section{CONCLUSIONS AND FUTURE WORK}

The 4SRS is a method organized in four main steps, that transforms UML use case models into systemlevel object diagrams, representing the software logical architectures of the system (Machado et al., 2006). The 4SRS method has already been used and tested in several real and complex projects as reported in (Ferreira et al., 2012; AAL4ALL, 2012).

In this paper, the 4SRS is adapted and extended in order to generate a data model supporting the resulting logical architecture. Herein, we start by distinguishing persistent data from non-persistent data allowing to create three new 4SRS steps with the aim of identifying entities, the relationships between entities and the entities' attributes.

The 4SRS table and the 4SRS first step (step 1) can be automatically generated from a set business process models, modeled in BPMN language, as presented in (Cruz et al., 2015). The use case descriptions comprise a set of structured and controlled sentences previously defined in (Cruz et al., 2014b). The generated use case descriptions can be complemented with other information every time it is necessary.

Integrating the approach presented herein and the approach proposed in (Cruz et al., 2015) it is possible to generate the use case model, software logical architectures and the data model based in a set of business process models. The derived software models can be used as bases to the development of the software that will support the business, helping to ensure the alignment between business process models and software models (Cruz et al., 2014a).

In a BPMN model, a sub-process may be used to describe the common part of different process models. Thus, when we are grouping a set of interrelated business process models, some use cases can appear more 
than once on the resulting use case model (Cruz et al., 2015). The 4SRS method is prepared to detect and eliminate duplicated use cases (Machado et al., 2006) (step 3) making it suitable for dealing with complexity. The generated models (use case model and data model) are consistent with each other, meaning that the data entities referred to in the use case model are represented in the data model.

As future work, we intend to apply this approach in a real industrial scenario which complexity and dimension will benefit from a systematic approach to the identification of the data model.

\section{ACKNOWLEDGEMENTS}

This work has been supported by FCT - Fundação para a Ciência e Tecnologia in the scope of the project: PEst-UID/CEC/00319/2013.

\section{REFERENCES}

AAL4ALL (2012). http://www.aal4all.org.

Bragança, A. and Machado, R. J. (2006). Extending UML 2.0 metamodel for complementary usages of the extend relationship within use case variability specification. In 10th International Software Product Line Conference (SPLC'06).

Chen, P. P.-S. (1976). The entity-relationship model toward a unified view of data. ACM Trans. Database Syst., 1:9-36.

Cockburn, A. (2001). Writing Effective Use Cases. Addison Wesley.

Cruz, E. F., Machado, R. J., and Santos, M. Y. (2014a). Derivation of data-driven software models from business process representations. In 9th International Conference on the Quality of Information and Communications Technology (QUATIC2014), pages 276281. IEEE-CS.

Cruz, E. F., Machado, R. J., and Santos, M. Y. (2014b). From business process models to use case models: A systematic approach. In Aveiro, D., Tribolet, J., and Gouveia, D. (Eds.), Advances in Enterprise Engineering VIII, vol. 174 of Lecture Notes in Business Information Processing, pages 167-181. Springer International Publishing.

Cruz, E. F., Machado, R. J., and Santos, M. Y. (2014c). On the decomposition of use cases for the refinement of software requirements. In Computational Science and Its Applications (ICCSA), 2014 14th International Conference on, pages 237-240. IEEE-CS.

Cruz, E. F., Machado, R. J., and Santos, M. Y. (2015). Bridging the gap between a set of interrelated business process models and software models. In 17th International Conference on Enterprise Information Systems, pages 338-345.
Fantechi, A., Gnesi, S., Lami, G., and Maccari, A. (2003). Applications of linguistic techniques for use case analysis. Requirements Engineering, 8(3):161-170.

Ferreira, N., Santos, N., Machado, R. J., and Gasevic, D. (2012). Derivation of process-oriented logical architectures: An elicitation approach for cloud design. In PROFES'2012, LNCS Series, Springer-Verlag, Berlin Heidelberg, Germany.

Ilieva, M. G. and Ormandjieva, O. (2006). Models derived from automatically analyzed textual user requirements. In Software Engineering Research, Management and Applications.

Machado, R., Fernandes, J. a., Monteiro, P., and Rodrigues, H. (2006). Refinement of software architectures by recursive model transformations. In Mnch, J. and Vierimaa, M. (Eds.), Product-Focused Software Process Improvement, volume 4034 of LNCS, pages 422-428. Springer Berlin Heidelberg.

OMG (2011). Business process model and notation (BPMN), version 2.0. Technical report, Object Management Group.

Samarasinghe, N. and Somé, S. S. (2005). Generating a domain model from a use case model. In Intelligent and adaptive systems and software eng.

Santos, M. Y. and Machado, R. J. (2010). On the derivation of class diagrams from use cases and logical software architectures. In 2010 5th Int'l Conf. on Software Engineering Advances.

Weske, M. (2012). Business Process Management Concepts, Languages, Architectures. Springer Science \& Business Media.

Yue, T., Briand, L., and Labiche, Y. (2009). A use case modeling approach to facilitate the transition towards analysis models: Concepts and empirical evaluation. In Schurr, A. and Selic, B., (Eds.) Model Driven Engineering Languages and Systems, volume 5795 of LNCS, pages 484-498. Springer Berlin Heidelberg.

Yue, T., Briand, L., and Labiche, Y. (2011). A systematic review of transformation approaches between user requirements and analysis models. Requirements Engineering, 16:75-99. 